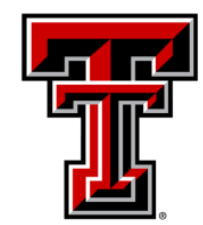

TEXAS TECH UNIVERSITY

Libraries"

\title{
A NEW FAST RESPONSE CRYOGENIC EVAPORATIVE CALORIMETER
}

\section{The Texas Tech community has made this publication openly available. Please share how this access benefits you. Your story matters to us.}

\begin{tabular}{|l|l|}
\hline Citation & $\begin{array}{l}\text { Andrew K. Gillespie, Cuikun Lin, Robert P. Thorn Jr., Heather } \\
\text { Higgins, Robert Baca, Andrew A. Durso, Django Jones, Ruth Ogu, } \\
\text { Jeremy Marquis, and R. V. Duncan, "A new fast response cryogenic } \\
\text { evaporative calorimeter", Review of Scientific Instruments 91, } \\
085103 \text { (2020) } \underline{\text { https://doi.org/10.1063/5.0013713 }}\end{array}$ \\
\hline Citable Link & $\underline{\text { https://hdl.handle.net/2346/87678 }}$ \\
\hline Terms of Use & C 2020 Author(s). \\
\hline
\end{tabular}




\section{A new fast response cryogenic evaporative calorimeter ${ }^{\circledR P}$}

Cite as: Rev. Sci. Instrum. 91, 085103 (2020); https://doi.org/10.1063/5.0013713

Submitted: 13 May 2020 . Accepted: 12 July 2020 . Published Online: 03 August 2020

(D) Andrew K. Gillespie, (D) Cuikun Lin, (D) Robert P. Thorn, Heather Higgins, Robert Baca, Andrew A. Durso, Django Jones, Ruth Ogu, Jeremy Marquis, and (D) R. V. Duncan

\section{COLLECTIONS}

This paper was selected as an Editor's Pick

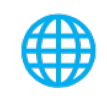

View Online

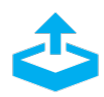

Export Citation

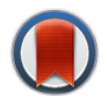

CrossMark

\section{ARTICLES YOU MAY BE INTERESTED IN}

An 18.3 MJ charging and discharging pulsed power supply system for the Space Plasma Environment Research Facility (SPERF). I. The overall design

Review of Scientific Instruments 91, 084702 (2020); https://doi.org/10.1063/5.0011711

Visible pump-mid infrared pump-broadband probe: Development and characterization of a three-pulse setup for single-shot ultrafast spectroscopy at $50 \mathrm{kHz}$

Review of Scientific Instruments 91, 073106 (2020); https://doi.org/10.1063/5.0016362

Design and performance of high-temperature furnace and cell holder for in situ spectroscopic, electrochemical, and radiolytic investigations of molten salts

Review of Scientific Instruments 91, 083105 (2020); https://doi.org/10.1063/1.5140463

\section{Challenge us.}

What are your needs for periodic signal detection?

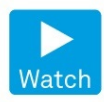

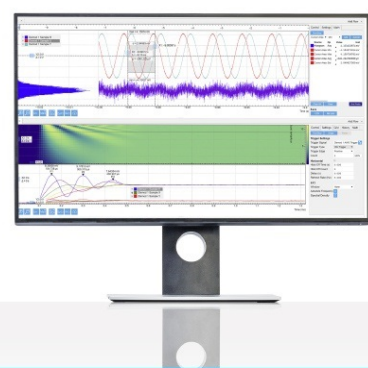




\title{
A new fast response cryogenic evaporative calorimeter
}

\author{
Cite as: Rev. Sci. Instrum. 91, 085103 (2020); doi: 10.1063/5.0013713 \\ Submitted: 13 May $2020 \cdot$ Accepted: 12 July 2020 • \\ Published Online: 3 August 2020
}

Andrew K. Gillespie,, a) (D) Cuikun Lin, 'D Robert P. Thorn, Jr., (D) Heather Higgins,' Robert Baca,

Andrew A. Durso,' Django Jones,' Ruth Ogu, Jeremy Marquis, ${ }^{2}$ and R. V. Duncan

\author{
AFFILIATIONS \\ ${ }^{1}$ Department of Physics and Astronomy, Texas Tech University, Lubbock, Texas 79409, USA \\ ${ }^{2} \mathrm{G}$ Systems, Inc., Richardson, Texas 75081, USA
}

a) Author to whom correspondence should be addressed: a.gillespie@ttu.edu

\begin{abstract}
We present the principle and implementation of a new type of fast response evaporative calorimeter designed to work at cryogenic temperatures and above-ambient pressures. It is capable of measuring input energy from an electric pulse and the thermal output energy by measuring the evaporation of liquid nitrogen through a mass flow meter. This system may be used to measure either the steady heat output from the system submersed under the cryogen or the heat output that results from a fast square-wave profile electrical pulse of duration from $10 \mu$ s or longer. The energy output of metal capillary-wire composite systems has been measured calorimetrically. A four-wire measurement was used to monitor the input electric energy with an uncertainty less than $5 \%$ for a typical pulse. Mass flow meters and pressure regulation systems were used to monitor the rate of evaporation of liquid nitrogen with a typical precision of $2 \mathrm{std}$.-ml/min. For a typical pulse, the integrated mass flow of nitrogen could be determined with an uncertainty less than $3 \%$. The pressure controllers and ballast compliance volumes allow the system to return to a steady state of mass flow in less than 2 min following an electric pulse. The system is capable of housing and measuring four separate wire-capillary systems in a single Dewar. On average, a calibration resulted in 3.9 std. ml evaporated per joule of input energy. This corresponds to a $97 \%$ efficiency for this calorimeter.
\end{abstract}

Published under license by AIP Publishing. https://doi.org/10.1063/5.0013713

\section{INTRODUCTION}

Calorimeters are useful analytic tools for quantitative analysis of exothermic and endothermic processes. In evaporative calorimetry, the heat evolved from energy changes is equated to the mass of gas boiled off from the surrounding calorimetric liquid. ${ }^{1-3}$ We report on a new type of evaporative calorimeter that is capable of measuring both the steady heat flow and the sudden pulsed heat-induced emissions from a cryogenic device under test (DUT). As in other such evaporative calorimeters, ${ }^{4-10}$ this system measured the heat from a device that was completely submersed within a cryogenic liquid so that the heat flow from the device became the latent heat of evaporation within the cryogenic liquid that completely surrounded the device. As in earlier systems, this measurement system can measure the steady emission of heat from the DUT. However, in addition, the system described here was capable of accurately measuring the sudden pulsed heat released from a DUT that was associated with electrical pulses, pulsed electromagnetic energy deposition, or triggered internal heat release from reactions, such as chemical or nuclear reactions.

Evaporative calorimeters were developed by the Los Alamos Scientific Laboratory in the late-1970s to measure the heat released from the products of nuclear fission reactions. ${ }^{5}$ These measurement systems utilized liquid helium as the cryogen, since liquid helium has a relatively small latent heat of evaporation, as detailed in Table I. This resulted in higher calorimetric sensitivity due to a relatively large flow of evaporated helium gas from a given amount of heat flow from the DUT (in this case, the byproducts from a nuclear reaction that was triggered by a thermal neutron pulse) into the liquid cryogen. When the temperature of the DUT is not a concern, then the performance of the evaporative calorimeter may be optimized by using a liquid cryogen with the smallest latent heat of evaporation available, which is typically liquid helium. However, if the operating temperature of the DUT is a concern or if the cost of the liquid cryogen becomes a factor in the design of the system, then less expensive cryogenic liquids, such as liquid nitrogen $\left(\mathrm{LN}_{2}\right)$, liquid argon, or 
TABLE I. Set point temperatures and the associated calorimetric sensitivity of evaporative calorimeters utilizing various cryogenic liquids. Note that the calorimetric sensitivities listed herein assume a flow-rate measurement accuracy of $0.1 \mathrm{std} .-\mathrm{ml} / \mathrm{s} .{ }^{13}$

\begin{tabular}{lcccc}
\hline \hline Liquid cryogen & $\begin{array}{c}\text { Boiling point temperature } \\
\text { at } \mathrm{P}=1 \text { atm }(\mathrm{K})\end{array}$ & Heat of vaporization $(\mathrm{J} / \mathrm{mol})$ & Evaporate vol. (std.-ml/J) & Calorimetric sensitivity $(\mu \mathrm{W})$ \\
\hline${ }^{3} \mathrm{He}$ & 3.2 & 24.6 & 911 & 0.11 \\
${ }^{4} \mathrm{He}$ & 4.2 & 82.6 & 271 & 0.37 \\
$\mathrm{H}_{2}$ & 20.3 & 882 & 25.4 & 3.9 \\
$\mathrm{Ne}$ & 27.1 & 1740 & 12.9 & 2.8 \\
$\mathrm{~N}_{2}$ & 77.4 & 5580 & 3.02 & 29 \\
$\mathrm{Ar}$ & 87.3 & 6450 & 3.47 & 30 \\
$\mathrm{O}_{2}$ & 90.2 & 6820 & 3.29 & \\
\hline
\end{tabular}

other liquids, may be utilized instead. These cryogens have a relatively wide liquid-vapor coexistence curve, so their liquid operating point temperature, which is the rejection temperature of the heat to be measured, is tunable over a wide range by adjusting the ambient pressure above the liquid cryogen. Equilibrium vapor pressure and temperature curves are published for all liquid cryogens, and composite information may be accessed through NIST's Thermophysics Division website. ${ }^{11}$

Typically, volume flow rates as low as $0.1 \mathrm{ml} / \mathrm{s}$ may be accurately measured using constant temperature anemometric flow meters. The power resolution of evaporative calorimeters is determined by dividing the measurement accuracy of the flow meter by the evaporated volume of the cryogen per joule of emitted heat from the DUT. Systematic errors, such as those discussed in Sec. II, usually dominate the uncertainty budget of the calorimeter and hence determine the actual energy and power accuracy of the calorimeter. The cryogen selected must operate at a pressure that is above its triple point pressure, and below the critical temperature, in order for the cryogen to be in its liquid state during the calorimeter's operation. The latent heat of vaporization of the liquid cryogen varies with the set point pressure of the calorimeter, and this goes to zero at the critical point of the cryogen. Hence, the operating pressure and the corresponding equilibrium temperature of the cryogen must be selected to be well below their critical values of the cryogen utilized for the calorimeter to operate properly. ${ }^{12}$ Table I displays the physical properties of liquid cryogens that may be used in the system design of evaporative calorimeters.

\section{SYSTEMATIC ERRORS ASSOCIATED WITH EVAPORATIVE CALORIMETERS}

If the entire DUT is surrounded with liquid cryogen such that the entire heat flow from the cryogenic object is fully converted to the evaporation of the liquid cryogen through its latent heat of evaporation, then the systematics associated with higher-temperature heat leaks into the calorimeter has only a minimal effect on the accuracy of the device since the physical readout of the evaporative calorimeter is simply the mass flow (not volume flow) of the vapor phase of the evaporated cryogen. Such a calorimeter is sensitive to any change in heat leaks directly to the liquid cryogen that occur after the device has been calibrated, as discussed in detail below. These variations have been minimized through the use of an annular guard tank of the same cryogen that intercepts the variations in heat leaks from the surrounding environment, as displayed in Fig. 1. Typically, the guard tank is operated near ambient pressure, and hence slightly colder than the inner experimental cryogen tank, which is typically held constant at about $5 \%$ over the ambient pressure levels. Furthermore, any oscillations of the vertical column of gas must be suppressed, and variations in radiative heat transfer down the neck of the device to the cryogen must be avoided to minimize systematic calorimetric errors, as described below. The design of the tests must assure that no evaporation results from splatter of the liquid cryogen onto the walls of the neck of the Dewar containing the DUT since that splatter evaporation will not be associated with heat flowing from the object itself. Finally, the pressure of the vapor over the cryogen must be held constant to a level that is adequate to avoid false indications that arise from the variations in the boil-off rate associated with ambient pressure changes over the liquid-cryogen bath. Atmospheric pressure variations may be as large as 5\% of the ambient atmospheric pressure as high- and low-pressure fronts (changes in the local weather) move through the vicinity of the evaporative calorimeter and as building air handling systems cycle on and off. The measurement system described here regulated the vapor pressure over the cryogenic liquid to one part in $10^{4}$ of its operating set point pressure, which was set to roughly $5 \%$ higher than the average ambient pressure.

When sudden pulsed release of heat occurs from the DUT, another significant systematic error may result due to the "piston effect," where some of the energy associated with the sudden heat release is converted into mechanical work, by momentarily lifting some mass of the cryogenic liquid against gravity, or by inducing mechanical flow of the liquid, or both. ${ }^{14-16}$ This effect becomes larger as the depth of the sudden heat release below the liquid-vapor surface becomes larger, and it potentially introduces a non-linearity into the measurement system since larger and faster heat releases may generate a larger fraction of mechanical work that is done on the cryogenic liquid. Examples of these accuracy-limiting effects, and methods to reduce them, are discussed in detail within this publication.

\section{DESCRIPTION OF DEVICE UNDER TEST}

We chose to examine the energy release from metal-hydride systems contained inside a pressurized stainless-steel capillary after 
(a)

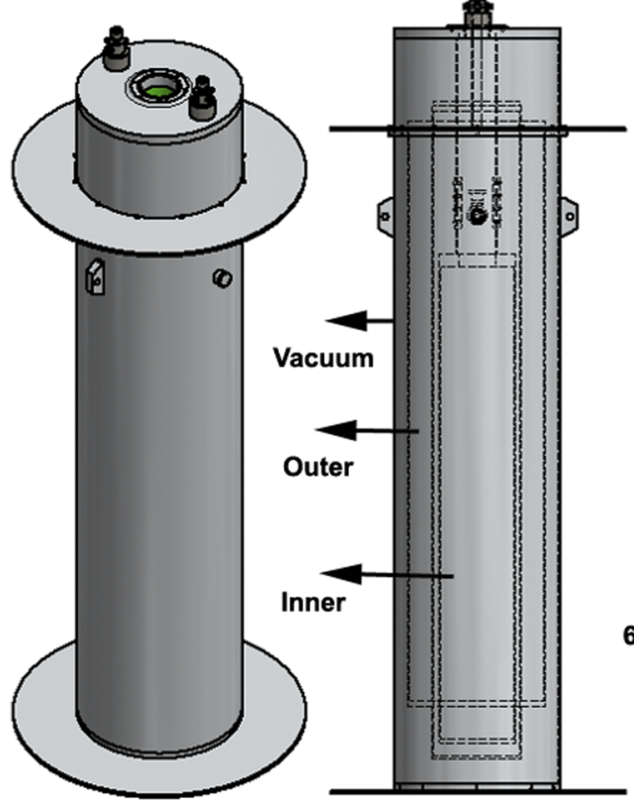

(b)

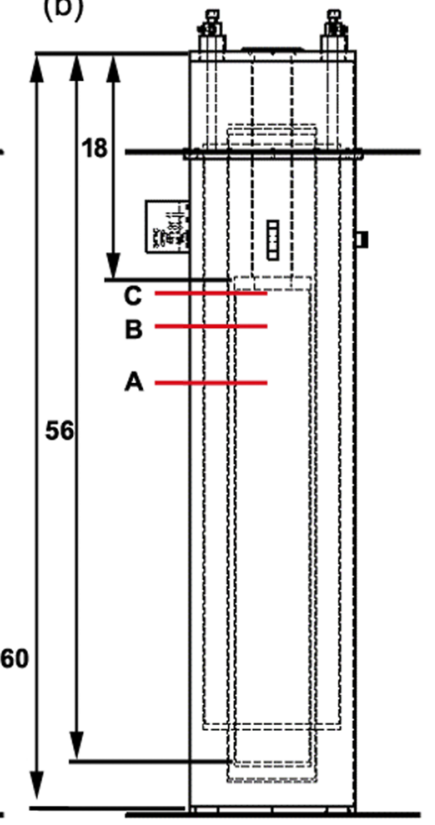

FIG. 1. Schematic of a two-stage Dewar that does not superheat. (a) There was a 37 I outer Dewar tank maintained near 700 Torr and an inner Dewar tank, which was actively regulated at a higher pressure typically near 712 Torr. While this Dewar system was typically operated using $\mathrm{LN}_{2}$, it was also tested with liquid argon and was designed to function well when liquid helium is used instead. (b) Three configurations for positioning the center of the DUT. Configuration $A$ was 8 in. below the Dewar neck. Configuration $B$ was 3 in. below the Dewar neck. Configuration $C$ was at the end of the Dewar neck. The total height of the two-stage Dewar is $60 \mathrm{in}$. The inner Dewar tank and Dewar neck have a combined height of 56 in. The Dewar neck is 18 in. in height. applying a square-wave electric pulse. The input energy was measured by monitoring the electric current and potential difference across the DUT. The output energy was measured by integrating the mass flow of evaporated nitrogen. This was accomplished by sealing the sample to be tested in $\mathrm{LN}_{2}$ inside a thermally isolated enclosure. The gas outlet was attached to Alicat mass flow meters (MW-5SLPM-D/CM, Alicat Scientific, Tucson, AZ) to monitor the flow of evaporated nitrogen. After subtracting a steady, very small baseline, the flow rate was integrated with respect to time, yielding the total amount of nitrogen gas that evaporated due to the sudden electric pulse.

The DUT was composed of a 6 in. metal wire placed in the middle of a stainless-steel capillary. This wire had a diameter of 0.009 in. and was made of palladium, platinum, or stainless steel, depending on the experiment. The capillary had an inner diameter of $0.0123 \mathrm{in}$. and an outer diameter of $0.0223 \mathrm{in}$. The wire-capillary system was curled in half such that the open ends of the capillary were in the same location above the top of the Dewar. In Fig. 2(a), the open ends of the capillary are located above the tube fitting. The capillary and four-wire probes were electrically isolated from the stainless-steel tube housing using Teflon tubing, Kapton tape, and an epoxy resin with a polymercaptan hardener (Loctite marine epoxy). The fourwire probes were separated from the capillary inside the T-fitting so that the wire-capillary system was hermetic and could be pressurized despite the feedthrough of the four-wire probes. These components were sealed in place near the t-fitting using marine epoxy. A manual bellows sealed valve from Swagelok Inc. was placed at the top where the DUT could be attached to a gas manifold for pressurization and pressure monitoring using an accurate manometer (8000 series TERPS, General Electric Energy, Measurement \& Control Solutions, Fairfield, CT). The capillary was pressurized using either protium or deuterium gas, depending on the experiment type.
The four-wire probes were twisted together to minimize electrical induction effects during pulsing. They were soldered to the capillary using a high silver content brazing alloy (Harris ${ }^{\circledR}$ Safety-Silv ${ }^{\circledR}$ 56 Silver Brazing Alloy, Harris Lincoln Electric, Mason, OH) using a hard-brazing technique. The metal-hydride system was contained in the middle of the capillary, which is located in the bottom 6 in. of the assembly between the brazed connections. The DUT was inserted straight down into the cryogen. The outside surface of the capillary was surrounded by nitrogen during these experiments. The small, higher resistance 0.010 in. diameter wires made of copper, manganese, and nickel alloy (Manganin 290, California Fine Wire, Grover Beach, CA) were used to monitor the potential difference across the capillary-wire system. The larger, 0.035 in. diameter copper wires with much less electrical resistance carried the pulsed current.

The 3/8 in.-diameter 304 stainless-steel tubing was used for housing the components. It was beneficial for the tubing to extend into the vapor space of the nitrogen Dewar, but definitely not into the liquid, in order to avoid heat variations from this tube into the $\mathrm{LN}_{2}$. The tubing kept the wires straight so that the vertical position of the 6 in. capillary-wire system could be accurately monitored. If the stainless-steel tubing extended into the liquid, it would conduct more heat into the system, increase the baseline mass flow rate, and increase the variation in that baseline mass flow rate. This can also cause an instability in the pressure of the inner Dewar, resulting in vapor oscillations within the column that must be suppressed in order to make accurate calorimetric measurements. If the liquid evaporated into the tube, the pressure inside the tube would be elevated relative to the Dewar pressure and vertical oscillation modes would ensue. Any variation of pressure in time would change the specific heat of the $\mathrm{LN}_{2}$, causing nonlinear behavior of the calorimeter's response. These oscillations could also shuttle heat from the 


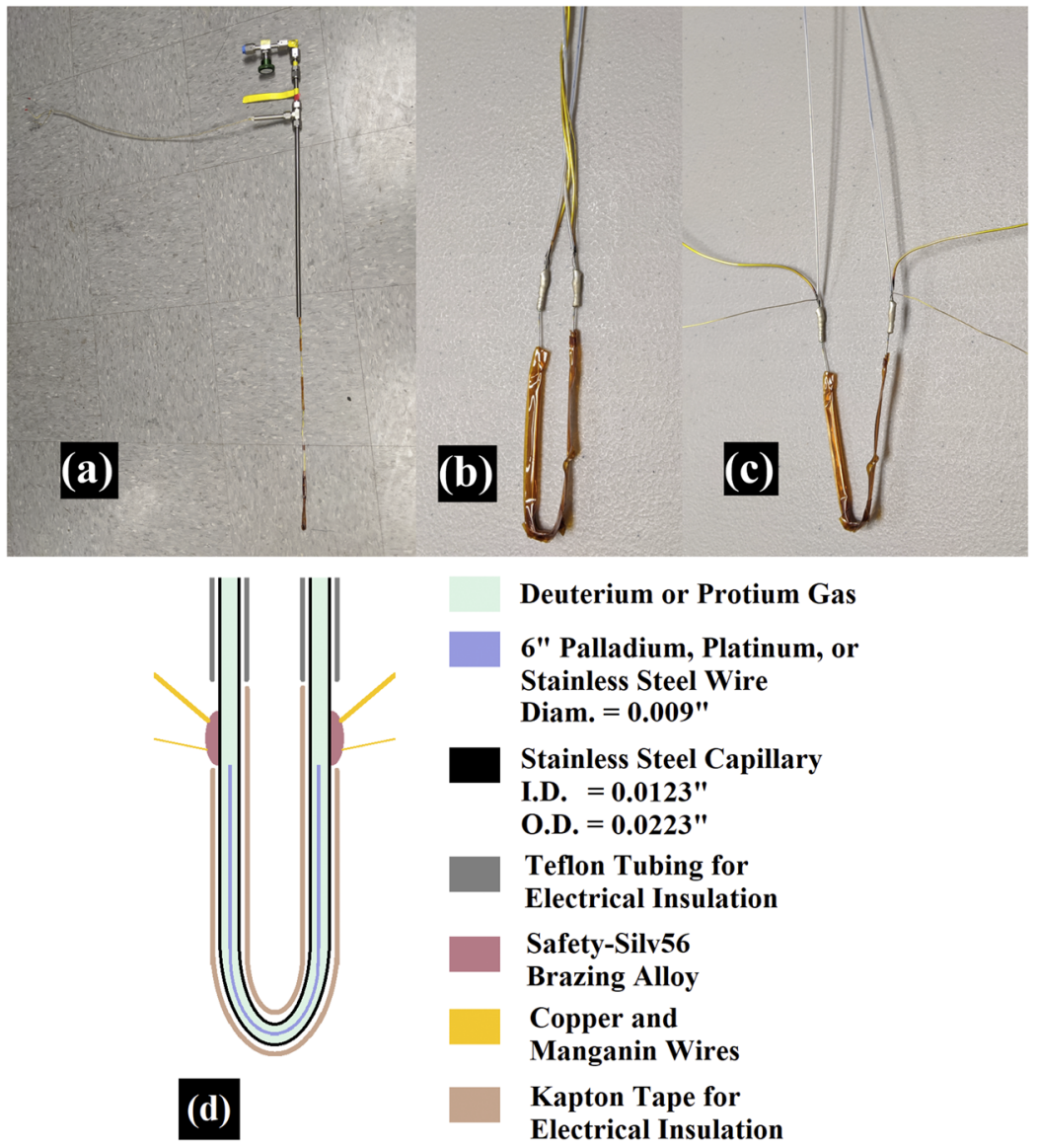

FIG. 2. The DUT was a wire-capillary system. (a) The entire DUT assembly with a manual valve, wire-capillary system, four-wire probes, stainless steel tubing for housing, and electric insulation. (b) A close-up image of the bottom 6 in. of the DUT assembly with electric insulation partially removed. (c) A second close-up image of the bottom 6 in. of the DUT assembly with the four-wire probes uncoiled from one another. (d) A diagram of the wire, capillary, electrical connections, and gases filling each volume when the DUT was submerged in the cryogen.
Dewar neck down into the $\mathrm{LN}_{2}$ layer, creating a false indication of evaporation from the sample under study.

\section{DESCRIPTION OF EVAPORATIVE CALORIMETER}

The calorimeter was composed of a pulsing system that measured the input electrical energy to the DUT and an automatic system that controlled the pressure stability over the liquid cryogen while measuring the mass flow of evaporated nitrogen gas. The custom-built pulsing system monitored the current and voltage at two locations to measure the energy of the outgoing pulse: (1) at the output terminals of the pulse generator and (2) at the connections to the DUT (Fig. 3). These two measurements were combined to infer the loss of pulse energy along the electrical leads, which was typically less than $4 \%$ of the total pulsed energy. In addition, the change in electrical energy that was stored in the line-isolated capacitor bank in the electric pulse generator was measured in order to bound the amount of energy delivered to the DUT. A programmable DC power supply (XR series, Magna-Power Corp. Flemington, NJ) was connected and used to charge the capacitor bank to between $200 \mathrm{~V}$ and $600 \mathrm{~V}$. Once charged, the capacitor bank was disconnected from the power supply to isolate the system from the external power grid. An insulated gate bipolar transistor (IGBT) switch was used to apply the pulse across the DUT. In this application, an IGBT switch was preferred over a high power bipolar junction transistor (BJT) or a metal-oxide-semiconductor field-effect transistor (MOSFET) because they combine greater power gain than the standard BJT with the higher voltage operation and lower input losses of the MOSFET. This highly robust IGBT was comprised of two parallel modules, each rated for $1700 \mathrm{~V}, 1200 \mathrm{~A}$, and $1 \mathrm{~ms}$. A four-gauge wire carried the current from the IGBT to an 18-gauge copper wire that was directly connected to the DUT. The potential drop across the DUT was monitored using high resistance 0.010 in. diameter manganin wires that were hard brazed to the capillary near the ends of the metal-hydride wire. The four-wire measurement allowed for separate, more precise measurements of current and voltage across the low resistance capillary system.

The applied pulse energy was measured from a separate board that incorporates both magnetic field and electrical potential drop as a means to determine instantaneous current output to the DUT. The current was monitored by measuring the voltage drop across a non-inductive, high frequency, coaxial shunt resistor from Powertek (A-2-01) with an accuracy of $0.2 \%$. A PEM Rogowski coil (CWT UM/30/B/1/80, GMW Associates, San Carlos, CA) was also used to inductively monitor the current as secondary confirmation of the resistive current measurement. The system was also capable of 


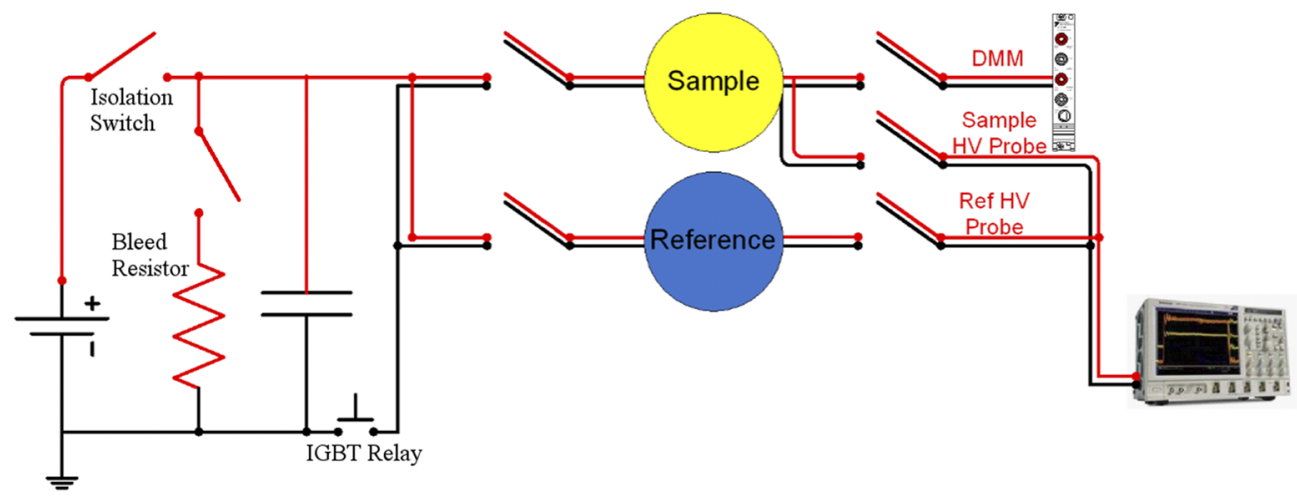

FIG. 3. Switch state diagram depicting the power supply, capacitor bank, bleed resistor, IGBT switch, wire test loads, digital multimeters, Tektronix oscilloscope (Tektronix, Beaverton, OR), and digitizer.

measuring the power dissipation in the pulse electronics board, in the leads, and in the DUT to quantitatively account for the total power output from the capacitors.

$$
E_{\text {Input }}=\int I V d t
$$

The potential drop across the wire-capillary system was integrated with respect to time along with the electric current to obtain the pulse energy. The energy uncertainty was determined using the $\pm 7 \mathrm{~V}$ uncertainty due to the high voltage probe, the $\pm 0.2 \%$ uncertainty in the current measurement from the shunt resistor, and the duration of the pulse. The input energy of a typical $31.5 \mathrm{~J}$ pulse could be determined with a $1.4 \mathrm{~J}$ uncertainty (Fig. 4).

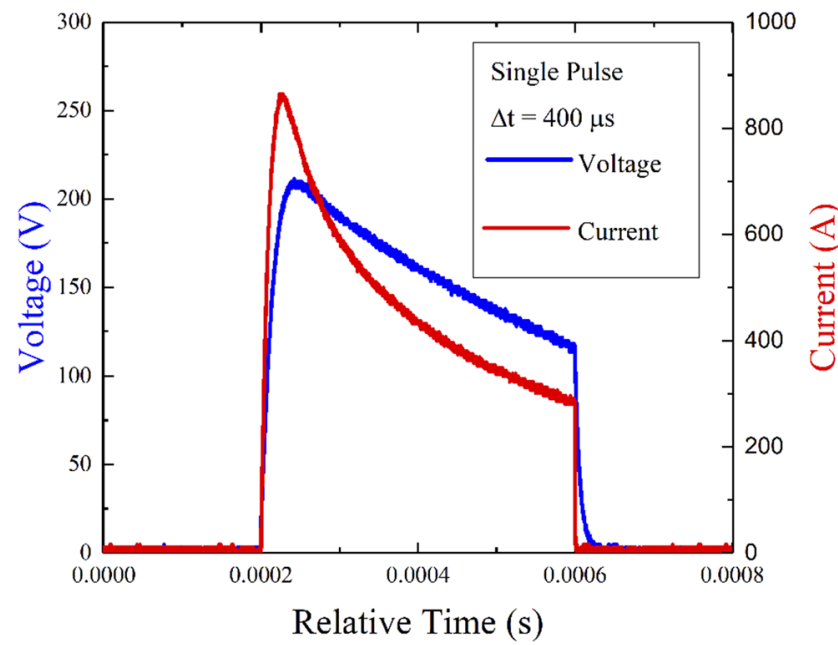

FIG. 4. The current and potential profiles as functions of time for a $31.5 \mathrm{~J}$ pulse across the DUT. The pulse width was $400 \mu \mathrm{s}$. Pulse widths varying from $10 \mu \mathrm{s}$ to $1.0 \mathrm{~ms}$ were measured using this system, with the fastest pulse width limited by the inductive time constant of the pulsed circuit, including the DUT. The resistance of the DUT varied with temperature and hence with time over the short duration of the pulse.
The capillary-wire system was submerged under $\mathrm{LN}_{2}$ contained inside the pressure system. All wires interfaced with the pressure system through electrically isolated, hermetic feedthroughs constructed from stainless-steel tubing and marine epoxy. The pressure system consisted of a Dewar, an Alicat mass flow meter, a pressure controller, and a ballast tank system used to improve the vapor pressure stability over the liquid cryogen during pulses.

The Dewar consisted of a 37-1, cylindrical shell outer $\mathrm{LN}_{2}$ tank. This outer tank was coaxial with a cylindrical inner tank that contains up to $15 \mathrm{l}$ of $\mathrm{LN}_{2}$. The outer tank connected mechanically and thermally to only one position along the neck of the inner tank, at $6 \mathrm{in}$. below the top flange. This intercepted most of the conductive heat coming down the epoxy-composite neck, so very little conducted heat entered the inner tank. More significantly, the outer tank was surrounded by vacuum space and intercepts all of the radiation heating from the room temperature shell of the Dewar. The outer tank, and the short aluminum shields that are directly cooled by the outer tank, completely surrounded the inner tank at every position except for the neck. Thus, none of this heat load was incident on the inner Dewar that was used for evaporative calorimetry. The inner Dewar was maintained at a slightly elevated pressure compared to the outer Dewar jacket so that a stable, very small amount of heat was radiated to the outer Dewar jacket. Thermal losses to the cryogenic liquid within the inner tank were exceptionally low and, if desired, could be made lossless by adjusting the differential operating pressures between the two tanks. The inner tank was designed with a rough surface to nearly eliminate superheating of the cryogen in the inner calorimeter tank during operation. The lack of bubble nucleation sites in Dewars with very smooth interior surfaces enables superheating. Hence, it is advantageous to use metal Dewar designs with rough edges, instead of glass Dewars, to avoid superheating. We have never observed any sudden, episodic increase in the boiling rate from the inner tank in over 250000 tank-hours of operation of this Dewar system, which was designed in collaboration with Richard Gummer of Precision Cryogenic Systems, Inc. (Indianapolis, IN).

It was critically important to regulate the pressure of the inner Dewar to achieve accurate evaporative calorimetry. The pressure regulation system held the vapor pressure over $\mathrm{LN}_{2}$ within the inner 
Dewar constant to within one part in 10000 . This degree of regulation was maintained so that the boil-off was not significantly biased by cryogen liquid temperature changes that were induced by atmospheric pressure variations. The outer $\mathrm{LN}_{2}$ tank was regulated to a constant set point pressure (and hence temperature) as well, though the exceptional thermal insulation of the inner and outer tank made this precaution unnecessary.

When the pressure of the nitrogen vapor changed in the Dewar, the temperature and specific heat of the $\mathrm{LN}_{2}$ also changed to keep the liquid in equilibrium with its saturated vapor pressure. Therefore, a predictable amount of heat flowed into or out of the liquid whenever the pressure was varied. If the pressure decreased, then the liquid cooled and the total heat capacity decreased, which required the liquid to expel heat. This occurred through evaporative cooling, so the $\mathrm{LN}_{2}$ evaporation rate increased as the ambient pressure decreased. The response time required for the liquid cryogen column to re-establish equilibrium should have been much shorter when the pressure decreased than when it increased. ${ }^{17}$ Since the liquid at the top of the column cooled when the pressure decreased, it became denser, creating an instability that resulted in convection within the $\mathrm{LN}_{2}$ column. So, only when it is cooled will the cryogen column freely convect to rapidly achieve thermal equilibrium. In contrast, if the pressure were increased, then the $\mathrm{LN}_{2}$ evaporation rate from the Dewar would decrease and the liquid at the vapor surface would warm. This would decrease its density, and the cryogen column would remain stable against convective onset. In this case, only thermal diffusion, which is a much slower process, would bring the column of the liquid cryogen into thermal equilibrium.

The change of the $\mathrm{LN}_{2}$ temperature with its vapor pressure is $0.0121 \mathrm{~K} /$ Torr near the ambient atmospheric pressure of 685 Torr in Lubbock, Texas. ${ }^{11,18,19}$ The specific heat of $\mathrm{LN}_{2}$ at this pressure and temperature is $2.046 \mathrm{~J} /(\mathrm{g} \mathrm{K})$, so the pressure-induced variation in the $\mathrm{LN}_{2}$ heat capacity is $(0.0121 \mathrm{~K} /$ Torr $) *(2.046 \mathrm{~J} / \mathrm{g} \mathrm{K})=0.0247$ $\mathrm{J} /$ (g Torr). $15 \mathrm{l}$ of $\mathrm{LN}_{2}$ in the inner tank had a mass of $12.1 \mathrm{~kg}$. Hence, the total heat that flowed in or out of the $\mathrm{LN}_{2}$ in the inner tank of the Dewar when it was full was $300 \mathrm{~J} /$ Torr. In addition, the inner tank was constructed from $5.79 \mathrm{~kg}$ of aluminum, specifically the AL 6061T6 alloy, which was in thermal equilibrium with the $\mathrm{LN}_{2}$ always, but effectively thermally disconnected from everything else, and which had a heat capacity at $\mathrm{LN}_{2}$ temperature of $340 \mathrm{~J} /(\mathrm{kg} \mathrm{K}) .^{20}$ Hence, the aluminum inner tank increased the total heat capacity by $7.9 \%$ above the heat capacity of the $\mathrm{LN}_{2}$, resulting in $324 \mathrm{~J} /$ Torr from heat into or out of the inner tank as the vapor pressure decreased or increased. This heat input or outflow in the $\mathrm{LN}_{2}$ was provided by the latent heat of evaporation or condensation, which was $199 \mathrm{~J} / \mathrm{g}$ at Lubbock's ambient pressure. As discussed above, any decrease in pressure would increase the nitrogen boil-off rate, and any increase in pressure would decrease it. In order to control the heat flow into or out of the inner tank to within a noise level of less than $25 \mathrm{~J}$, it was necessary to regulate the pressure of the nitrogen vapor within the Dewar to within $10 \mathrm{~Pa}$ of its set point pressure. Hence, the pressure of the inner Dewar had to be regulated to this degree throughout the entire time that they were in use as calorimeters. Figure 5 shows the typical pressure fluctuation prior to the electrical pulsing of the DUT.

When regulated, the Dewar pressure typically stayed within $10 \mathrm{~Pa}$ of the set point for a minimum of $5 \mathrm{~h}$ before measurements

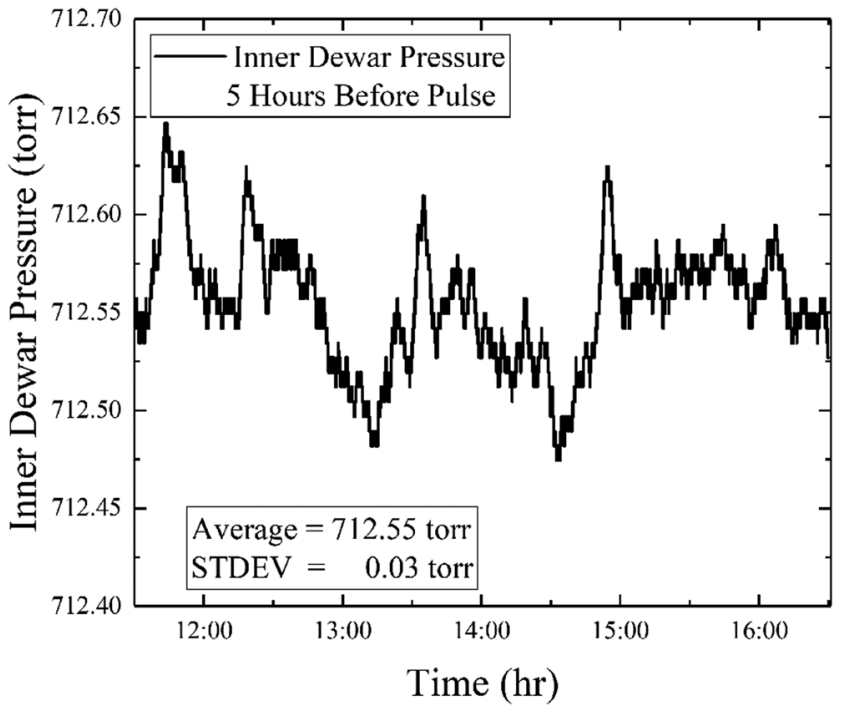

FIG. 5. The pressure stability while the Dewar was in a steady flow state. The pressure was maintained at 712 Torr, above the ambient pressure of 685 Torr, and there was a constant flow of nitrogen out of the pressure system of about 60 std.$\mathrm{ml} / \mathrm{min}$ as the liquid evaporated. The pressure stability was typically below \pm 0.05 Torr, or approximately one part in 10000 .

were made, resulting in an average power noise over this period prior to the measurements of about $2 \mathrm{~mW}$. This gave adequate time for the Dewar boil-off to achieve a stable baseline prior to making calorimetric measurements. An $8 \mathrm{~mW}$ increase in power dissipation within the inner tank would be required to produce a change of $2.0 \mathrm{std} .-\mathrm{ml} / \mathrm{min}$ in the flow rate of nitrogen out of the Dewar, which was typically the noise level of the baseline in this exceptionally stable system. Hence, $8 \mathrm{~mW}$ was typically the minimum resolution of this evaporative calorimeter, and the regulation of the Dewar pressure to within $10 \mathrm{~Pa}$ over a 5-h period assured that pressure would not produce a systematic error above the noise level of the calorimeter.

In designing the regulation system to keep the pressure constant to $10 \mathrm{~Pa}$ within the inner tank, it was necessary to assure that any sudden flow of nitrogen boil-off that occurred when the DUT was pulsed electrically would not create an enduring pressure change within the Dewar that would violate the level of isobaric conditions within the nitrogen and hence disturb the evaporative calorimeter at the levels discussed above. It was also important that these electrical pulses did not drive the pressure regulating servo unstable at the level of $10 \mathrm{~Pa}$ or higher for an extended time period.

This pressure surge within the inner tank was measured during routine pulsing using a very stable and accurate 8000 series high precision and high stability GE TERPS pressure sensor, while the resulting flow of nitrogen boil-off was measured using various capacity Alicat Whisper series mass flow meters and different methods of pressure regulation. The TERPS pressure sensor is shown connected to the Dewar cap in Fig. 6, along with the four wires that connect to the pulse generator circuitry. The Alicat PCD15PSIA-D dual valve differential pressure regulator was found to be lower in pressure noise and faster in pulse pressure recovery in this 


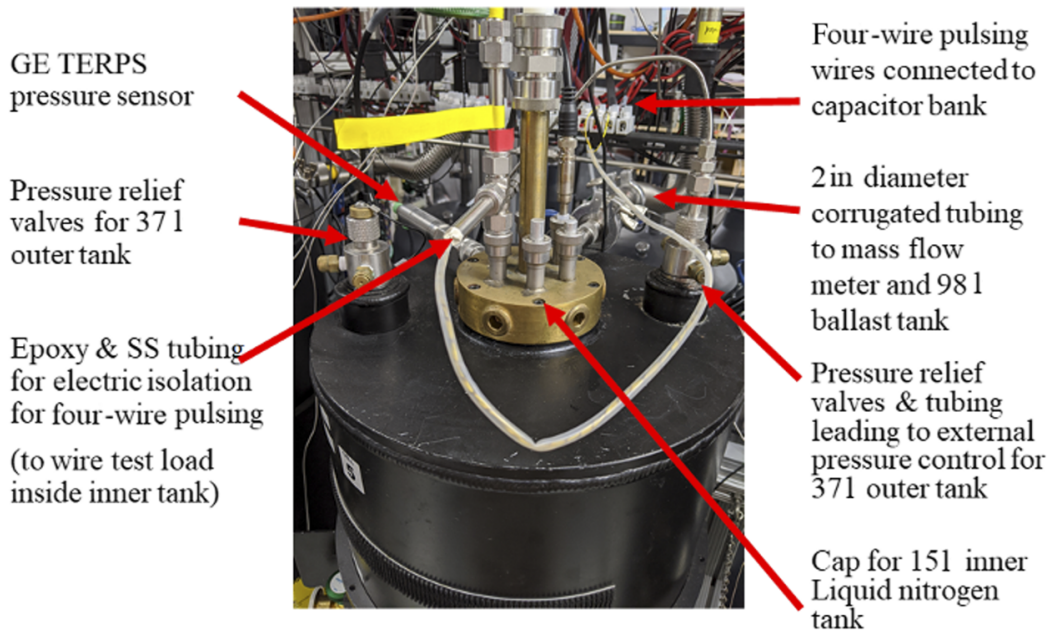

FIG. 6. The Dewar, pressure sensor, pressure relief valves, four-wire pulse probes, tubing to ballast tanks, and a single DUT inserted vertically into the inner Dewar tank. specific application than the MKS Baratron capacitance manometer Model 244 and MKS Baratron-based servo-controlled Model 216 pressure regulator valve (MKS Instruments, Andover, MA). The compliance volume above the liquid must exceed $100 \mathrm{l}$ to assure that the pressure surge during the pulse would not adversely affect the pressure stability of the ballast. This ballast volume was provided by connecting a 981 thermally insulated tank to the inner tank using large 2 in. diameter corrugated stainless steel tubing. Another $11 \mathrm{l}$ ballast tank was connected in series to the $98 \mathrm{ltank}$ using $1 / 8$ in. stainless steel tubing, which improved the flow stability (Figs. 7 and 8 ).

The Alicat 5 SLPM (maximum range) mass flow meters had an adequately low flow impedance with a pressure drop of $400 \mathrm{~Pa}-$ $4000 \mathrm{~Pa}$ to avoid unacceptable pressure surges during pulsing. They also provided adequate mass flow measurement accuracy to measure the boil-off flow rate from the inner tank to the ballast volume. To provide adequate pressure regulation in the ballast tank, a cascaded regulation system was used. We incorporated an ultrafast response "belch function" that immediately released $99.5 \%$ of the measured sudden flow into the ballast within $2 \mathrm{~s}$ of a pulse in order to bring the pressure regulation back to steady-state conditions in the ballast much more rapidly than the ballast volume servo could provide acting alone. The total, nearly instantaneous flow into the ballast tank, immediately after a pulse applied to the DUT, was measured by integrating the flow from the Dewar to the ballast using the Alicat mass flow meter's integration function. Then, within $2 \mathrm{~s}$, the same volume of nitrogen to within $\pm 0.5 \%$ was released from the ballast using a programmable flow meter

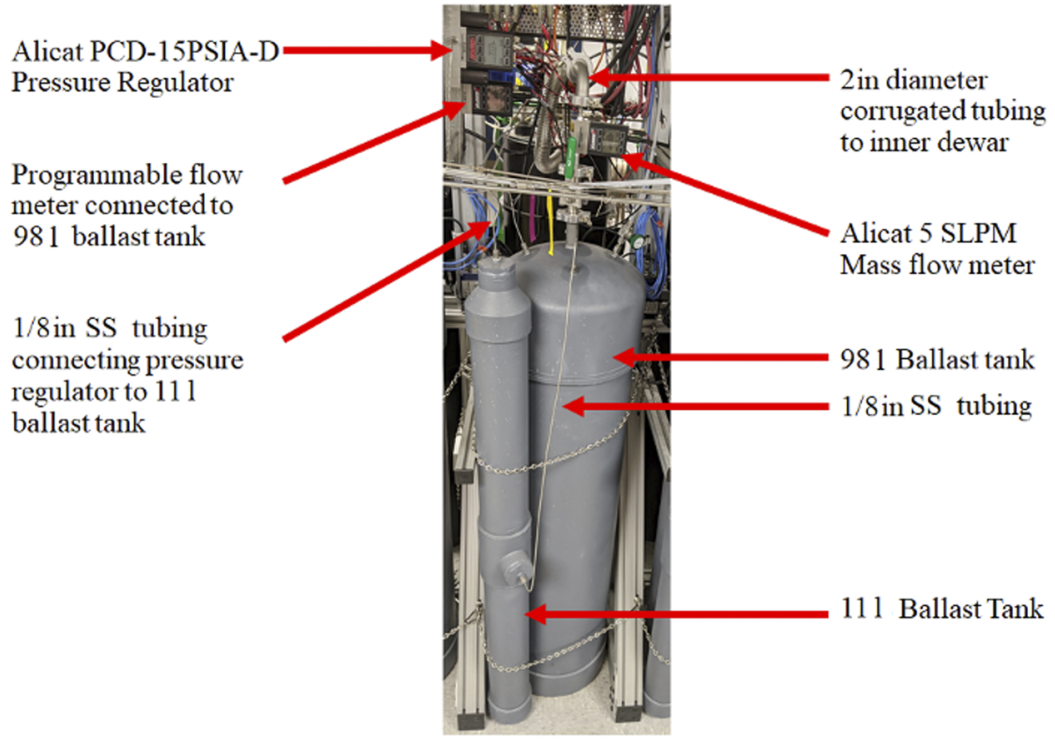

FIG. 7. The ballast tanks, flow meters, and pressure controllers used to maintain a steady state of mass flow. 


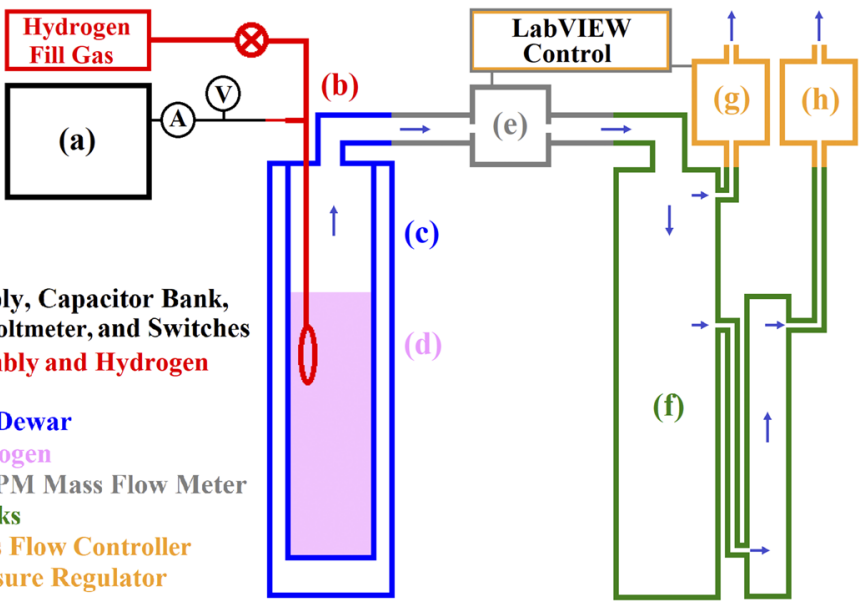

FIG. 8. A simplified diagram of the liquid and pneumatic systems. The capacitor bank was connected to the wires of the DUT. The DUT was inserted into the two-stage Dewar and was submerged in liquid nitrogen. When liquid was evaporated, it flowed through the 2 in. corrugated, stainlesssteel tubing, through the 5 SLPM mass flow meter, and into the ballast volumes. The programmable mass flow meter ejected $99.5 \%$ of the integrated mass flow to assist the fast convergence of the pressure while the pressure regulator maintained the vapor pressure.

and proportioning valve on the ballast volume's exhaust port. This system assured that the pressure servo that regulated the pressure within the ballast would quickly converge and not experience prolonged damped oscillations as it converged back to its set point. This cascade system maintained the pressure of the inner tank to within $10 \mathrm{~Pa}$ of its set point pressure for an indefinite period of time.

The mass flow system was capable of measuring the evaporated gas from the Dewar due to the pulse. A steady baseline mass flow was recorded and averaged before the pulse was administered. The output energy was determined by integrating the baseline-subtracted nitrogen mass flow over the 2-min period immediately following an individual electric pulse. For example, a typical pulse with an input energy of $31.5 \pm 1.4 \mathrm{~J}$ produced a nitrogen boil-off of 124 \pm 3.7 std.-ml (Fig. 9). The uncertainty of the excess boil-off due to the pulse was estimated based on the measured stability of the baseline boil-off rate.

We implemented an integrated system that automatically calibrates the boil-off of the $\mathrm{LN}_{2}$ as a function of the input pulse energy, given the dissipation pulse-vs-time profile, the thermal heat exchange properties of the DUT, and the depth profile of the DUT below the $\mathrm{LN}_{2}$ surface. This resulted in a system that may use $\mathrm{LN}_{2}$ level sensing and DUT positioning to predict the anticipated calibration slope of the calorimeter. A significant component of the instrument was the software written using LabVIEW for data acquisition, data logging, and analysis. A custom software application was written by G-Systems, Inc. to acquire, process, analyze, and present data in an automated fashion. This automation coordinated the flow and pressure control to stabilize the Dewar and to detect and minimize any systematic errors.

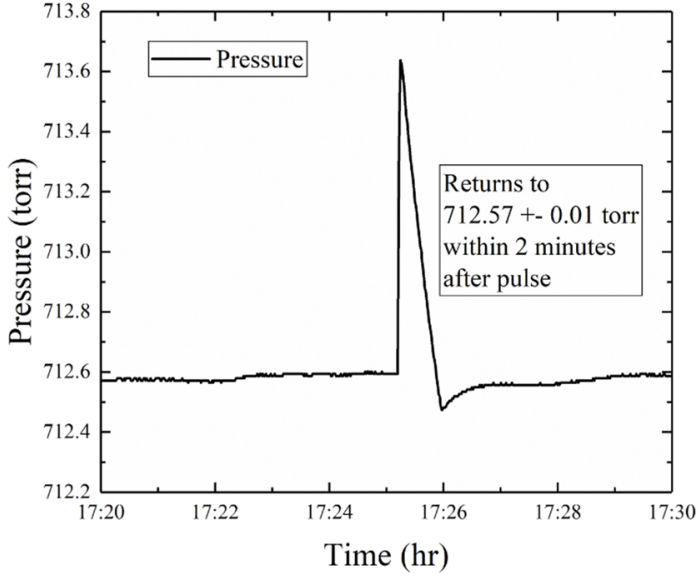

(a)

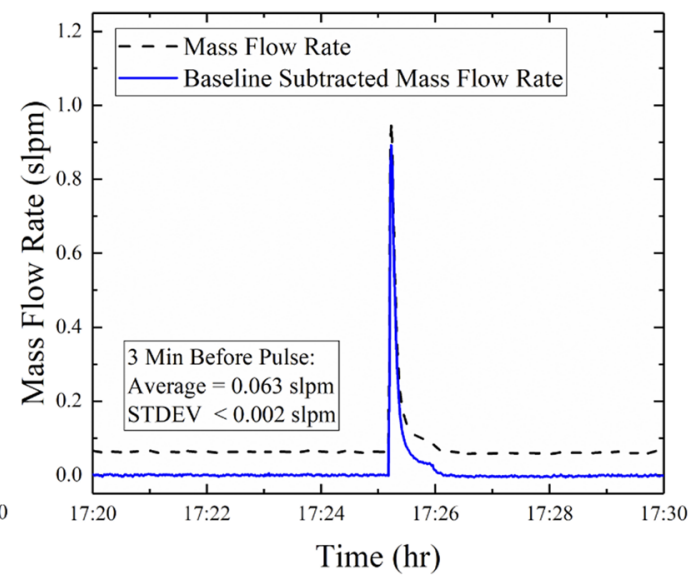

(b)

FIG. 9. (a) The pressure variation in time and (b) the mass flow variation in time before, during, and after a typical electric pulse on a DUT described in Fig. 2(d). The pressure regulation system and ballast compliance volume regulated the pressure such that it converged without experiencing prolonged oscillations. 


\section{CALIBRATION AND MEASUREMENTS}

Reference and sample wires were connected to the four-wire pulse probes using a solder connection. Each reference and sample wire were placed in the Dewar such that the soldered joint was submerged under the liquid-vapor interface. The system was sealed, and then the pressure and mass flow were monitored. After the pressure and mass flow reached a steady state and had been regulated for more than $5 \mathrm{~h}$, the system could be calibrated by determining the total nitrogen boil-off mass as a function of the input energy. A stainless-steel reference wire was pulsed first using input energies that typically varied by a factor of three. To ensure a stable nitrogen boil-off flow rate, the Dewar pressure and mass flow were monitored for 3 min prior to administering each pulse. The standard deviation in the baseline flow rate was used in estimating the uncertainty of the output mass flow. The pulse was administered to the reference wire, which underwent joule heating. After subtracting the steady baseline, the mass flow was integrated from a time just before the pulse was administered until $2 \mathrm{~min}$ after the pulse. This process was repeated several times using varied input energies to create a linear calibration trend.

The experiment in Fig. 10 resulted in a calibration slope of 4.07 $\pm 0.05 \mathrm{std} .-\mathrm{ml} / \mathrm{J}$ when the solder connections were positioned $3.6 \mathrm{in}$. below the liquid-vapor interface. Since the 6 in. capillary-wire system spanned 3 in. vertically in the Dewar, the average position of the pulsed wire was $5.1 \mathrm{in}$. below the liquid-vapor interface. If the DUT simply underwent joule heating, then it would boil off an equal amount of nitrogen and coincide with the calibration trend from the reference. An exothermic reaction within the DUT would result in a larger amount of boil-off while an endothermic reaction within the DUT would boil off less nitrogen.

The latent heat of vaporization of $\mathrm{LN}_{2}$ is $199 \mathrm{~J} / \mathrm{g}$, and the density of nitrogen is $1.25 \mathrm{~g} / \mathrm{l}$ at STP. This corresponds to $4.02 \mathrm{std} .-\mathrm{ml} / \mathrm{J}$ at STP. ${ }^{21,22}$ The calibration slope from this experiment was $101 \%$ of the value at STP, which means that this calorimeter obtained $100 \%$ efficiency within the uncertainty when pulsing under these conditions.

The wire-capillary system spanned a vertical distance of 3 in. in the liquid. When the average cell position was 0 in., the solder connections were located 1.5 in. above the liquid-vapor interface and the bottom curve in the wire-capillary system was located $1.5 \mathrm{in}$. below the liquid-vapor interface. The calorimeter was typically used with the wire-capillary system positioned in configuration $\mathrm{A}$, which was 8 in. below the Dewar neck, as seen in Fig. 1(b). The system was used over the course of several days in one configuration. Though the configuration of the DUT was not changed, $\mathrm{LN}_{2}$ evaporated at a steady rate, resulting in a slow change in the submersion depth with time. When pulsing the DUT with the wire-capillary system located close to the liquid-vapor interface, the average calibration slope was near $3.9 \mathrm{std} .-\mathrm{ml} / \mathrm{J}$ in accordance with what is predicted from the latent heat of vaporization (Fig. 11). This corresponds to a $97 \%$ efficiency for this calorimeter. However, the calibration slope was diminished if the capillary-wire system was pulsed more than 5.7 in. below the liquid-vapor interface due to a "piston effect," as discussed in Sec. VI. This effect became larger as the depth of the sudden heat release below the liquid-vapor surface became larger. The piston effect potentially introduced a non-linearity into the measurement system since more intense heat releases may have generated a larger

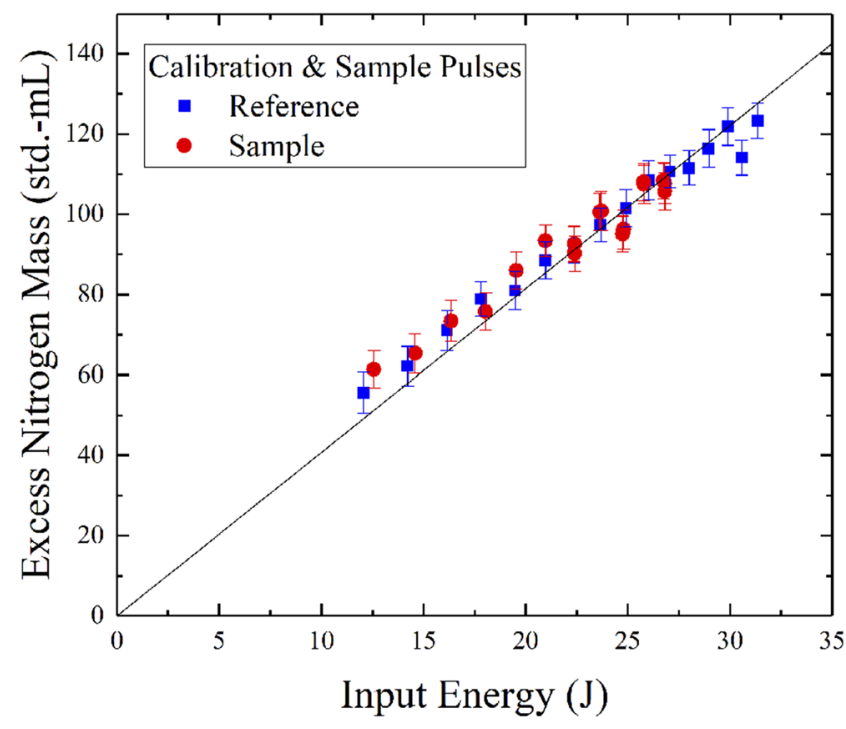

FIG. 10. Excess nitrogen mass that boiled off as the result of the input energy of the electric pulses of a reference and sample wire. The stainless-steel reference wire was pulsed over a wider range of input energies, shown in blue. The relationship between the excess nitrogen mass and the input pulse energy provided a linear calibration for which to compare the sample performance. The sample DUT was pulsed over a smaller range of input energies, shown in red.

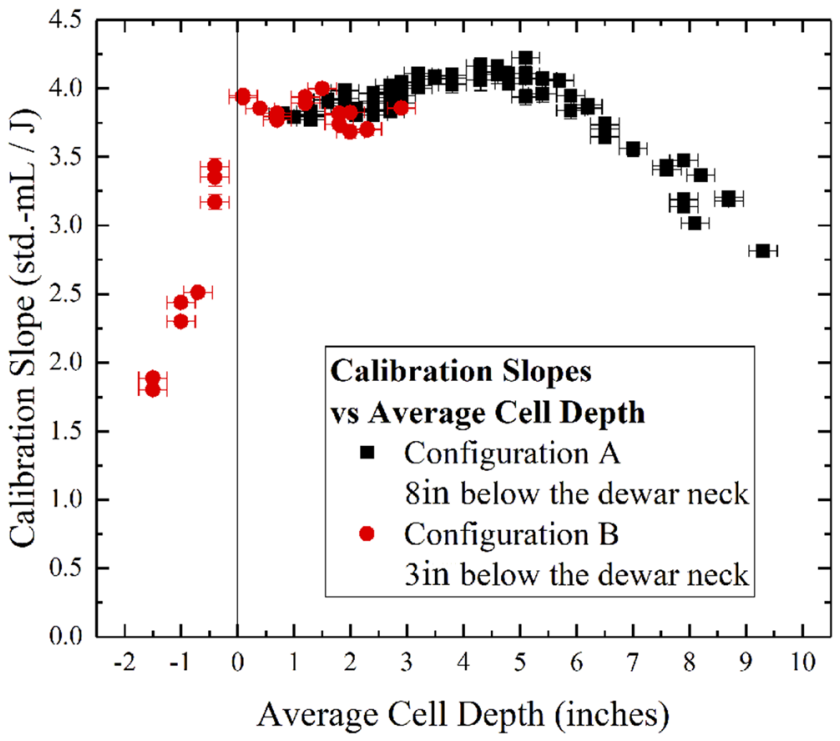

FIG. 11. Calibration slope as a function of submersion depth of the average position of the sample cell. The DUT was pulsed with the wire-capillary system located at least 3 in. below the Dewar neck in configuration A or B. The sensitivity of the calorimeter decreased with depth as energy was transferred into momentum of the liquid, dissipating slowly out of the system. The sensitivity also decreased when the wire-capillary system was located above the liquid-vapor interface. 
fraction of mechanical work done on the cryogenic liquid. A fraction of the pulsed energy was converted into work, first against gravity as the liquid mass rose, and then into mechanical flow once the mass of nitrogen fell back down. Since the total energy is conserved, the calibration slope was diminished with increased depths as more of the energy went into producing mechanical work. This mechanical flow in the liquid cryogen eventually dissipated, but the flow persisted for a longer length of time than the measurement window following the pulse. Hence, this mechanical flow was not attributed with the dissipation from the sudden pulse of electrical energy.

When pulsing the DUT with the wire-capillary system located above the liquid-vapor interface, the calibration slope was also diminished. As a smaller fraction of the 6 in. wire-capillary system was submerged, less input energy was imparted to the liquid. This resulted in less evaporation of the $\mathrm{LN}_{2}$ as a fraction of the input energy simply heated the vapor space. The mass flow meter only measured the evaporated mass, so the energy used to heat the vapor was lost from the calorimeter measurements.

When pulsing the DUT with the wire-capillary system located near the neck of the Dewar at location C, systematic errors may occur (Fig. 12). The Dewar neck conducted heat into the system at a greater rate than the inner Dewar tank. If $\mathrm{LN}_{2}$ splashed onto the Dewar neck as a result of an electric pulse, then a larger amount of nitrogen would be evaporated by the small amount of heat traveling down the Dewar's neck, feigning an exothermic reaction within the liquid cryogen. This could potentially result in higher calibration slopes or non-linear behavior in the measured mass flow for a given input energy.

Splashing during pulsing caused greater variation in the calibration slope. A fiber-optic-based camera was introduced into the Dewar neck through a hermetic seal on the top of the Dewar flange and focused to observe any splashing that occurred during pulsing.

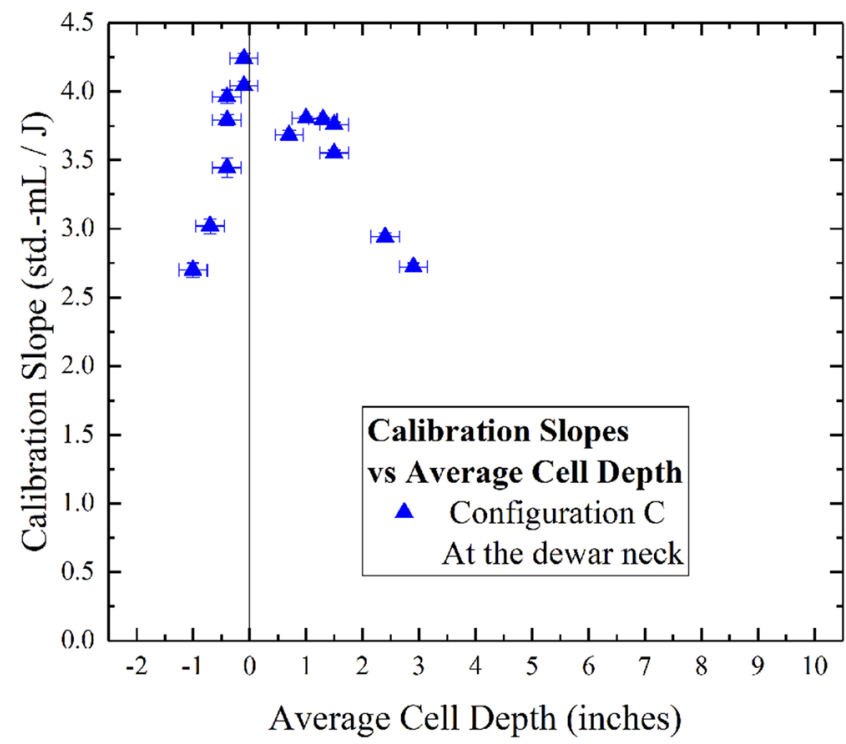

FIG. 12. Calibration slope as a function of submersion depth of the average position of the sample cell. The DUT was pulsed with the wire-capillary system located near the Dewar neck in configuration $\mathrm{C}$.
Splashing was more likely to occur when the soldering joint was close to the liquid-vapor interface. In our original sample cell design, the solder joint was located at or near the liquid-vapor interface near the Dewar neck. We increased the overall length of our sample cells to reduce splashing. After the design change, the DUT was pulsed below the Dewar neck in configuration A with the solder connection fully submerged. This resulted in less systematic errors and a higher degree of linearity since the boil-off was solely due to the input pulse energy and not heat transfer from splashing onto the Dewar neck.

\section{THERMOPHYSICS OF PULSED EVAPORATIVE CALORIMETRY}

Steady-state heat transfer from the DUT to the liquid cryogen provided a rate of cryogen boil-off that was easily predicted from the latent heat of evaporation of the cryogen. However, when the DUT was suddenly pulsed, fast transient effects occurred. Some of the electrical energy that created the sudden pulse would actually be converted into mechanical work on the cryogen, either by momentarily elevating part of the cryogen column against gravity as the evaporation occurs, or by inducing mechanical flow of the cryogen, or both. While these effects would eventually produce additional thermal dissipation as the cryogen's mechanical motion relaxed, this relaxation would often take more time than the time window that is allotted to the evaporation measurement directly associated with the pulse. Given the noise in the baseline evaporation rate, as discussed above, some slow dissipation of this resulting mechanical motion would fall below this baseline noise level and would not be directly associated with the pulse. Hence, this conversion of some of the energy within the rapidly expanding vapor would be converted to mechanical work, in a process that we refer to as the "piston effect." The size of this piston effect strongly depended upon the time rate of change of the heat flowing out from the DUT into the cryogen and on the position profile of the DUT below the liquid-vapor surface. The nature and measurements of these variations is the topic of this section. Suffice to say that the calorimeter must be calibrated for the actual configuration and depth profile of the DUT and the range of pulse durations that are employed experimentally. While pulsing the 6 in. wire-capillary system in our DUT, the piston effect was only significant when the system was located more than 5.7 in. below the liquid-vapor interface. Pulsing below that depth increased the piston effect and decreased the calorimeter efficiency, as seen in Fig. 11.

As the depth of the heat dissipated under the liquid-vapor surface of the cryogen increased, there was a larger mass of cryogen above the point of dissipation that could be placed into mechanical motion due to the piston effect. This mass was momentarily elevated in its gravitational potential before it fell back down to induce mass flow within the liquid. In our simple model, we assumed that the sudden increase in the cryogen's potential energy above the point of dissipation fully converted into mechanical flow energy as the liquid fell back to its original height. Hence, as the depth of the sudden heat dissipation increased, the cryogenic liquid above that point acted to directly convert some of this dissipated heat in the DUT into mechanical motion.

Any energy that is converted into kinetic mixing and goes undetected by the mass flow meters will manifest as a decrease in 
calorimeter efficiency. This kinetic energy of mixing from an individual pulse may be approximated by comparing the integrated mass flow with the calibration slope when the calorimeter is operating near $100 \%$ efficiency. In the absence of the piston effect, the calibration slope should be near $4.02 \mathrm{std}$.- $\mathrm{ml} / \mathrm{J}$, as predicted from the latent heat of vaporization of $\mathrm{LN}_{2}$. The kinetic energy of mixing may be approximated as

$$
\Delta K E=\frac{\Delta m_{\mathrm{out}}}{\lambda_{\mathrm{LN} 2}}=E_{\mathrm{in}}-\frac{m_{\mathrm{out}}}{\lambda_{\mathrm{LN} 2}},
$$

where $\Delta m_{\text {out }}$ is the difference between the integrated mass flow and that expected upon the latent heat of evaporation (100\% calorimetric efficiency), $\lambda_{\mathrm{LN} 2}=4.02 \mathrm{std} .-\mathrm{ml} / \mathrm{J}$, and $m_{\text {out }}$ is the integrated mass flow from an individual pulse of input energy $E_{\text {in }}$. Alternately, this could be represented in terms of the calibration slope from a set of measurements over a span of input energies as

$$
\Delta K E=E_{\text {in }}\left[1-\frac{S_{(\mathrm{i})}}{\lambda_{\mathrm{LN} 2}}\right],
$$

where $S_{(\mathrm{i})}$ is the calibration slope when pulsing at a specific depth below the $\mathrm{LN}_{2}$ interface. The amount of energy converted into kinetic mixing is a simple percent of the input energy, as represented by a ratio of the calibration slope to $4.02 \mathrm{std}$.- $\mathrm{ml} / \mathrm{J}$. As an example, if this DUT were pulsed $8.5 \mathrm{in}$. below the liquid-vapor interface, one would expect the calibration slope near $3.0 \mathrm{std} .-\mathrm{ml} / \mathrm{J}$ due to the piston effect in accordance with Fig. 11. If pulsed with an input energy of $20 \mathrm{~J}$, the integrated mass flow would be near 60 std. $-\mathrm{ml}$ with a calorimetric efficiency near $75 \%$. An energy of $15 \mathrm{~J}$ was immediately converted to evaporate the detectable mass flow and $5 \mathrm{~J}$ would raise the column of the cryogen and be converted into kinetic mixing.

It may be useful to calculate how high the cryogen is elevated during a typical pulse when the piston effect is present. One may approximate the mass of liquid above the point of dissipation as a cylinder with a diameter of the inner Dewar tank. Therefore, momentarily elevating the mass will store gravitational potential energy as

$$
U_{\mathrm{g}}=m g \Delta h=\left[\rho \pi R^{2} d\right] g \Delta h,
$$

where $R$ is the radius of the inner Dewar tank, $d$ is the depth at which the pulsed energy is being dissipated, and $\Delta h$ is the elevation height. This energy is converted into kinetic mixing of the cryogenic fluid. If this potential energy is converted into kinetic mixing, then Eq. (3) may be equated to Eq. (4) to yield the elevation height as a function of depth below the liquid-vapor interface. In other experiments, various thicknesses of a thin layer of a thermally insulating material called polyimide was placed on the outer surface of the stainless steel capillary to limit the maximum rate of heat transfer to the liquid and hence to limit the piston effect. While this strategy was marginally successful, such coatings introduce additional complexity. The calibration procedures described above are recommended to quantitatively account for calorimetric inefficiencies that result from the piston effect,

$$
\Delta h=\frac{E_{\mathrm{in}}\left[1-\frac{S_{(\mathrm{i})}}{\lambda_{\mathrm{LN} 2}}\right]}{\left[\rho \pi R^{2} d\right] g} .
$$

If the DUT were pulsed in the same manner discussed above with an input energy of $20 \mathrm{~J}$, then the $75 \%$ efficiency would correspond to
$5 \mathrm{~J}$ raising the liquid above the point of dissipation by $1.2 \mathrm{~mm}$. If the DUT were pulsed under the same conditions as the pulse in Figs. 4 and 9 , then the $31.5 \mathrm{~J}$ with a $97 \%$ efficiency would correspond to $0.7 \mathrm{~J}$ raising a much smaller mass of the liquid above the point of dissipation by only $0.2 \mathrm{~mm}$. The geometries of the DUT and the inner Dewar tank place constraints on the operation of this calorimeter. This shows that for the calorimeter to operate at $100 \%$ efficiency, immersion depth must be taken into consideration.

\section{DISCUSSION OF FAST AND SLOW PROCESSES}

Due to the high voltages and currents used in this work, a typical pulse had a maximum power input greater than $50 \mathrm{~kW}$. These experiments resulted in a calorimeter efficiency near 97\%. This shows that the calorimeter is applicable for fast processes involving large power inputs. The calorimeter was tested using small power input as well. A DC power supply was attached to the DUT to supply a constant current of 3.00 A to the DUT. A four-wire measurement was used to monitor the potential difference across the wirecapillary system during the pulse. The potential difference across the capillary-wire system was constant at $0.86 \pm 0.02 \mathrm{~V}$ during these pulses. The low power input was dissipated directly into the $\mathrm{LN}_{2}$ without substantial temperature rise within the DUT, so the temperature of the wire-capillary system remained only slightly higher than the surrounding temperature of the cryogenic liquid during heat transfer. Therefore, the resistance of the wire-capillary system remained constant to within measurement uncertainties, resulting in a square-wave pulse profile and no piston effect. The input power was constant at $2.6 \pm 0.1 \mathrm{~W}$. The DUT was pulsed when the average position of the wire-capillary system was $5.7 \mathrm{in}$. below the liquid-vapor interface in configuration A.

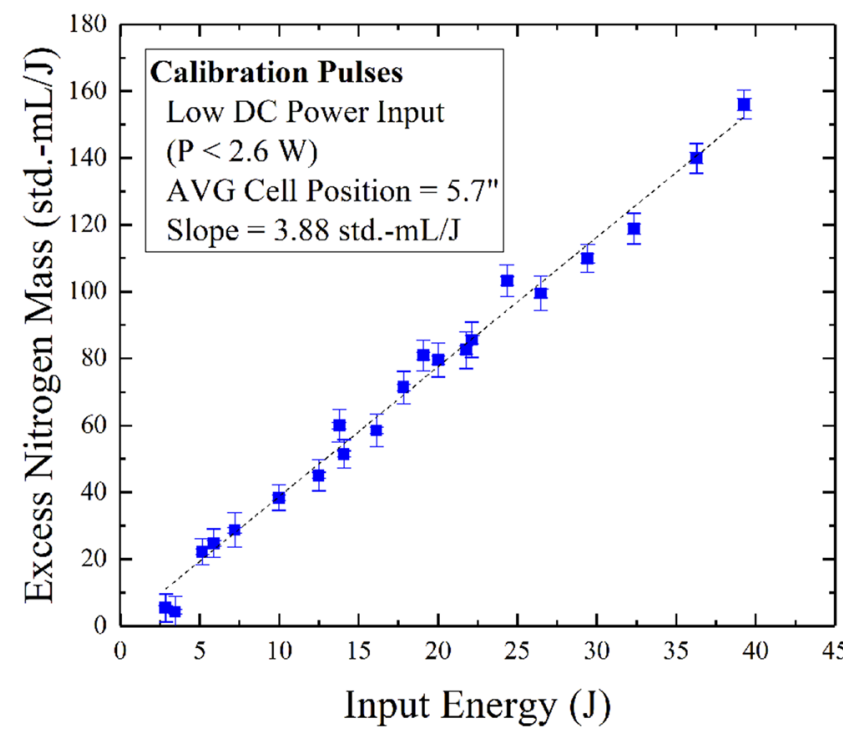

FIG. 13. Excess nitrogen mass vs input energy for low power input, square-wave pulses to the DUT with the average position of the system $5.7 \mathrm{in}$. below the liquidvapor interface in configuration $A$. 
The input energy was varied by changing the duration of the square-wave pulse from $0 \mathrm{~s}-15 \mathrm{~s}$ so that the input energy spanned a similar range compared to the energy used when measuring fast processes. After subtracting the steady baseline, the mass flow rate was integrated to obtain the excess nitrogen mass flow. The calibration slope was $3.88 \mathrm{std}$.- $\mathrm{ml} / \mathrm{J}$, which corresponded to a calorimeter efficiency of $97 \%$ (Fig. 13). This confirms that the calorimeter operates with the same efficiency regardless of whether a fast, highinput power process is measured or whether a slow, low-input power process is measured by the evaporative calorimeter.

\section{DISCUSSION OF POWER DISSIPATION}

The system can monitor the power dissipation within all components leading to the DUT. The capacitor bank was charged and then disconnected from the grid by disconnecting a physical switch from the power supply. Isolating the system in this manner prevented any major energy delivery errors. This also sets an upper bound on the amount of energy that could be delivered to the DUT.

The energy change across the capacitor bank was monitored using a voltage probe. A second voltage probe was used to monitor the potential difference across the DUT. Once the capacitor bank was charged and isolated, the upper bound for energy stored in the capacitor can be calculated by

$$
E_{0}=\frac{1}{2} C \Delta V^{2} \text {, }
$$

where $C$ is the total capacitance and $\Delta V$ is the potential difference across the capacitors. An IGBT switch was used to apply the pulse across the DUT. The energy delivered by the capacitor to the external circuit, including the leads, IGBT, and DUT was determined by integrating the current with the potential difference across all components, where $V_{1}$ and $V_{2}$ are the electric potential differences, as displayed in Fig. 14,

$$
E_{1}=\int I V_{1} d t
$$

The energy delivered to the capillary-wire system, excluding the leads, IGBT switch, and current carrying wires, was determined by integrating the current with the potential difference across the 6 in. capillary-wire system alone,

$$
E_{2}=\int I V_{2} d t
$$

Note that $E_{0}>E_{1}>E_{2}$. This allows one to quantitatively account for the total power output from the capacitors.

The capacitor bank was charged and administered a pulse to a resistor four separate times. Each pulse used the same duration

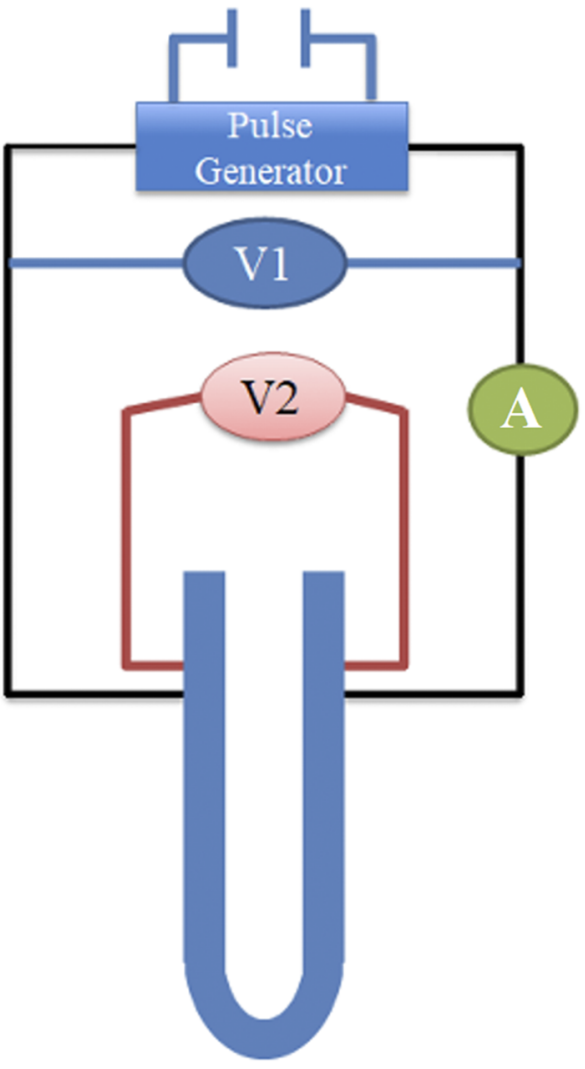

FIG. 14. Simplified wiring diagram of the pulse generator system and an external circuit that includes two voltmeters, one ammeter, and the device under test. One voltage probe was used to monitor the potential difference across the pulse generator. A second voltage probe monitored the potential difference across the DUT.

of $50 \mu \mathrm{s}$, and the charge voltage was varied from $400 \mathrm{~V}$ to $700 \mathrm{~V}$. As detailed in Table II, an average of about $4 \%$ of the total pulse energy was dissipated in the pulse generator, and an additional $4 \%$ was dissipated by the external circuit leading to the device under test. Approximately $92 \%$ of the pulsed energy was dissipated in the DUT. Depending on the experiment, it may become important to track the energy dissipated external to the calorimeter. However, only the energy dissipated in the DUT contributes to the evaporation of the cryogenic fluid. If the input energy is being reported as the energy dissipated across the DUT, then any energy dissipated in external

\begin{tabular}{|c|c|c|c|c|c|}
\hline Capacitor voltage (V) & $\mathrm{E}_{0}(\mathrm{~J})$ & $\mathrm{E}_{1}(\mathrm{~J})$ & $\mathrm{E}_{2}(\mathrm{~J})$ & $\begin{array}{l}\text { Energy dissipated in pulse } \\
\text { generator }(\mathrm{J})\end{array}$ & $\begin{array}{c}\text { Energy dissipated in circuit } \\
\text { leading to DUT }(\mathrm{J})\end{array}$ \\
\hline 400 & 8.77 & 8.45 & 8.06 & $0.32(3.7 \%)$ & $0.39(4.4 \%)$ \\
\hline 500 & 13.16 & 12.32 & 11.76 & $0.84(6.4 \%)$ & $0.56(4.2 \%)$ \\
\hline 600 & 17.23 & 16.64 & 15.94 & $0.59(3.4 \%)$ & $0.70(4.1 \%)$ \\
\hline 700 & 21.65 & 21.03 & 20.20 & $0.61(2.8 \%)$ & $0.83(3.9 \%)$ \\
\hline
\end{tabular}
components does not affect the calorimetric results.

TABLE II. Energy dissipation in components of the pulsing system. The pulse duration was $50 \mu \mathrm{s}$ for each pulse with varied capacitor charge voltage. 


\section{CONCLUSIONS}

Our cryogenic calorimeter allowed for the detection of heat output for pulsed wire systems within $10 \%$ certainty, or better, depending on the experimental configuration. This new type of fast response evaporative calorimeter was capable of measuring input energy from an electric pulse with an uncertainty less than $5 \%$ for a typical pulse. For a typical pulse, the integrated mass flow of nitrogen could be determined with an uncertainty less than $3 \%$. The calibration slope was constant near 3.9 std.-ml/J when the DUT was fully submerged up to a depth of 5.7 in., after which the calibration slope decreased due to the piston effect. This calibration slope was commensurate with the heat of vaporization of $\mathrm{LN}_{2}$. This calorimeter may be used to measure power dissipation and conversion to heat energy over a wide range of time scales. While electrical pulses were applied to a sample immersed in $\mathrm{LN}_{2}$ in this work, the same principles may be applied to measure heating that results from other forms of energy deposition, such as that from particle beams or other sources and within other cryogenic liquids.

\section{ACKNOWLEDGMENTS}

The authors would like to thank Dr. Richard Gummer, Dr. Stephen Bayne, Dr. James A. Schrock, Duy K. Nguyen, Sarah Seger, Emily Hirsch, and the Texas Tech physics shop for their technical assistance and useful discussions. The authors would like to acknowledge G Systems, Inc., for providing programming, assembly, and design services while under contract to Texas Tech on this effort. This work was supported by the Texas Research Incentive Program and by Texas Tech University. The identification of commercial products, contractors, and suppliers within this article is for informational purposes only and does not imply endorsement by Texas Tech University, their associates, or their collaborators.

\section{DATA AVAILABILITY}

The data that support the findings of this study are available from the corresponding author upon reasonable request.

\section{REFERENCES}

${ }^{1}$ W. P. Gilbreath and D. E. Wilson, Rev. Sci. Instrum. 41, 969 (1970).

${ }^{2}$ R. O. Williams, Rev. Sci. Instrum. 36, 1441 (1965).

${ }^{3}$ C. A. Reading and A. Reiser, J. Phys. E: Sci. Instrum. 10, 1069 (1977).

${ }^{4}$ E. Calvet, Les Développements Récents de La Microcalorimétrie et de La Thermogenèse, 156th ed. (du Centre National de la Recherche Scientifique, Paris, 1967).

${ }^{\mathbf{5}} \mathrm{P}$. Bendt and J. Yarnell, in Cryogenic Engineering Conference, CONF-770801-2, Boulder, CO, USA, 1977.

${ }^{6}$ J. Yarnell and P. Bendt, Los Alamos Scientific Laboratory Informal Report Nos. NUREG/CR 0349 and LA-7452-MS, USA, 1978.

${ }^{7}$ G. Coletta, L. Gherardi, and F. Gömöry, IEEE Trans. Appl. Supercond. 9(2), 1053 (1999).

${ }^{8}$ F. Darmann, S. Dou, and C. Cook, IEEE Trans. Appl. Supercond. 13, 1 (2003).

${ }^{9}$ P. T. Eubank, J. C. Holste, L. E. Cediel, D. H. Moor, and K. R. Hall, Ind. Eng. Chem. Fundam. 23, 105-109 (1984).

${ }^{10}$ J. P. Murphy, M. J. Mullins, P. N. Barnes, T. J. Haugan, G. A. Levin, M. Majoros, M. D. Sumption, E. W. Collings, M. Polak, and P. Mozola, IEEE Trans. Appl. Supercond. 23(3), 4701505 (2013).

${ }^{11}$ E. Lemmon, M. McLinden, and D. Friend, NIST Chemistry WebBook, NIST Standard Reference Database Number 69, edited by P. J. Linstrom and W. G. Mallard (National Institute of Standards and Technology, Gaithersburg, MD, 2018), http://webbook.nist.gov.

${ }^{12}$ J. E. Fesmire, Phys. Procedia 67, 1089-1097 (2015)

${ }^{13}$ F. Pobell, Matter and Methods at Low Temperatures, 3rd ed. (Springer-Verlag Berlin Heidelberg, 2007).

${ }^{14}$ D. Cross, B. Hughes, D. Ingham, and L. Ma, Tunnelling Underground Space Technol. 61, 71-81 (2017).

${ }^{15}$ F. A. McDonald and G. C. Wetsel, J. Appl. Phys. 49, 2313 (1978).

${ }^{16}$ B. Auvity, M. Bellenoue, and T. Kageyama, Exp. Fluids 30, 221-228 (2001).

${ }^{17}$ M. Belmedani, A. Belgacem, and R. Rebiai, J. Appl. Sci. 8, 2544-2552 (2008).

${ }^{18}$ J. Jensen, W. Tuttle, R. Stewart, H. Brechna, and A. Prodell, "Properties of nitrogen," in Brookhaven National Laboratory Cryogenic Data Handbook (Brookhaven National Laboratory, New York, 1980), Sec. VI, https://www.bnl.gov/magnets/staff/gupta/cryogenic-data-handbook/Section6.pdf. ${ }^{19}$ A. Van Itterbeek, A. de Bock, and L. Verhaegen, Physica 15, 624 (1949).

${ }^{20}$ P. Duthil, "Material properties at low temperature," in Proceedings of the CERN Accelerator School, CAS 2013 (Publisher: CAS - CERN, Italy, 2013), pp. 77-95.

${ }^{21}$ G. White, Experimental Techniques in Low Temperature Physics, Monographs on Physics and Chemistry of Materials, 3rd ed. (Clarendon Press, Oxford, 1979).

${ }^{22}$ S. Van Sciver, "Low-temperature materials properties," in Helium Cryogenics (Springer, Boston, MA, 1986). 